\title{
A Logic for Reasoning about Knowledge of Unawareness*
}

\author{
Thomas Ågotnes and Natasha Alechina
}

\begin{abstract}
In the most popular logics combining knowledge and awareness, it is not possible to express statements about knowledge of unawareness such as "Ann knows that Bill is aware of something Ann is not aware of" - without using a stronger statement such as "Ann knows that Bill is aware of $p$ and Ann is not aware of $p$ ", for some particular $p$. In Halpern and Rêgo (2006, 2009b) (revisited in Halpern and Rêgo (2009a, 2013)) Halpern and Rêgo introduced a logic in which such statements about knowledge of unawareness can be expressed. The logic extends the traditional framework with quantification over formulae, and is thus very expressive. As a consequence, it is not decidable. In this paper we introduce a decidable logic which can be used to reason about certain types of unawareness. Our logic extends the traditional framework with an operator expressing full awareness, i.e., the fact that an agent is aware of everything, and another operator expressing relative awareness, the fact that one agent is aware of everything another agent is aware of. The logic is less expressive than Halpern's and Rêgo's logic. It is, however, expressive enough to express all of the motivating examples in Halpern and Rêgo (2006, 2009b). In addition to proving that the logic is decidable and that its satisfiability problem is PSPACE-complete, we present an axiomatisation which we show is sound and complete.
\end{abstract}

Thomas Ågotnes

University of Bergen, e-mail: thomas.agotnes@infomedia.uib.no

Natasha Alechina

University of Nottingham, e-mail: natasha.alechina@nottingham.ac.uk

* A preliminary version of this paper appeared in Ågotnes and Alechina (2007). This paper is published in the Journal of Logic, Language and Information June 2014, Volume 23, Issue 2, pp 197-217. 
Thomas Ågotnes and Natasha Alechina

\section{Introduction}

Formal models of knowledge or belief extended with a notion of awareness has been of interest to researchers in several fields, including economics and game theory, philosophy, and multi-agent systems. One of the most popular frameworks is the logic of general awareness (Fagin and Halpern, 1988), which has been shown (Halpern, 2001) to be a generalisation of frameworks used by economists (Modica and Rustichini, 1994, 1999). The logic of general awareness has a traditional (implicit) knowledge operator $K_{i}$ where $K_{i} \phi$ is interpreted as truth of $\phi$ in all accessible worlds in a Kripke structure, in addition to an awareness operator $A_{i}$ where $A_{i} \phi$ is interpreted by a syntactic assignment of truth value, and an explicit knowledge operator $X_{i}$ such that $X_{i} \phi$ is interpreted as the conjunction of $K_{i} \phi$ and $A_{i} \phi$. This framework is very flexible and general. However, as pointed out by Halpern and Rêgo (2006), in many situations, agents have knowledge about their own or others' unawareness, and this cannot be expressed properly in the logic of general awareness. An example, taken directly from (Halpern and Rêgo, 2006), is the following.

Example 1.

Consider an investor (agent 1) and an investment fund broker (agent 2). Suppose that we have two facts that are relevant for describing the situation: the NASDAQ index is more likely to increase than to decrease tomorrow (p), and Amazon will announce a huge increase in earnings tomorrow (q). [...] [B]oth agents explicitly know that the NASDAQ index is more likely to increase than to decrease tomorrow. However, the broker also explicitly knows that Amazon will announce a huge increase in earnings tomorrow. Furthermore, the broker explicitly knows that he (broker) is aware of this fact and the investor is not. On the other hand, the investor explicitly knows that there is something that the broker is aware of but he is not.

In order to be able to reason formally about situations involving knowledge of unawareness such as this one, Halpern and Rêgo (2006, 2009b) introduced a logic which extends the logic of general awareness with variables standing for formulae and quantification over these variables. For example, the formula $X_{1}\left(\exists x\left(A_{2} x \wedge \neg A_{1} x\right)\right)$ expresses the fact that the investor, in the example above, explicitly knows that there is some fact he is unaware of but the broker is aware of. This introduction of quantifiers makes the logic very expressive, but unfortunately also makes it undecidable.

There is a subtle distinction in the motivating arguments of Halpern and Rêgo (2006, 2009b). On the one hand, it is initially argued that it would be useful to express the fact that an agent "knows that there are facts of which he is unaware". We will refer to awareness of everything as full awareness. Explicit knowledge of the lack of full awareness can be expressed in Halpern's and Rêgo's logic by a formula such as $X_{i}\left(\exists x \neg A_{i} x\right)$. On the other hand, Example 1 above requires the expression of knowledge of a more specific property of unawareness: that an agent (explicitly) knows that he is unaware of some fact which another agent is aware of. We will refer to this latter form of unawareness as lack of relative awareness. We say that an agent has relative awareness with respect to another agent if he is aware of everything the 
other agent is aware of. As discussed above, knowledge of lack of relative awareness can be expressed in Halpern's and Rêgo's logic by a formula such as $X_{i}\left(\exists x\left(A_{j} x \wedge\right.\right.$ $\left.\neg A_{i} x\right)$ ). Full awareness implies relative awareness, but in general not the other way around.

The logic proposed by Halpern and Rêgo (2006, 2009b) was criticised by researchers working on formal models of awareness in mathematical economics, where the approach to modelling awareness and the assumptions made are somewhat different. In particular, two common assumptions are that awareness is generated by primitive propopositions (agpp), and that agents know what they are aware of $(\mathrm{ka})$. Given these assumptions, the statement that an agent does not know whether he is aware of all formulas, $\neg X_{i} \neg \forall x A_{i} x \wedge \neg X_{i} \forall x A_{i} x$, is not satisfiable in the logic of Halpern and Rêgo $(2006,2009 \mathrm{~b})$, so such an agent cannot be modelled in the logic. To solve this problem, a new variant of the logic is introduced in Halpern and Rêgo (2009a, 2013), where each 'possible world' has a different language associated with it.

In this paper we introduce an alternative logic for reasoning about knowledge of unawareness, which extends the logic of general awareness with explicit operators for full and relative awareness. For each agent $i$, the logic has a nullary operator $C_{i}$ standing for "agent $i$ has full awareness", and for each agent $i$ and each agent $j$ a nullary operator $R_{i j}$ standing for for "agent $j$ has greater awareness relative to agent $i$ '. In this language, both types of knowledge of unawareness mentioned above can be expressed, viz. as $X_{i} \neg C_{i}$ and $X_{i} \neg R_{j i}$, respectively. With these operators in place of unlimited quantification over formulae, the logic is, obviously, much less expressive than Halpern's and Rêgo's logic. However, it can be used to express all the motivating examples in (Halpern and Rêgo, 2006, 2009b). Furthermore, the logic presented in this paper is decidable, as other epistemic modal logics developed in computer science are, and can be used for automated reasoning and verification. The property describing an agent being uncertain whether he is aware of all formulas, which motivated the development of Halpern and Rêgo (2009a, 2013), is expressible in the preliminary version of our logic (Ågotnes and Alechina, 2007) as $\neg X_{i} \neg C_{i} \wedge \neg X_{i} C_{i}$. It is satisfiable without the resort to having different languages in different possible worlds. However, in (Ågotnes and Alechina, 2007) we did not consider the agpp and $k a$ assumptions. With those assumptions added, the property of being uncertain concerning the awareness of all formulas is no longer satisfiable. It was pointed out by Halpern and Rêgo (2013) that they believe that the variant of our logic presented in (Ågotnes and Alechina, 2007) can be modified so that it does not have this problem and is still decidable, but they have not checked this conjecture. We show that this is indeed the case, by incorporating both the agpp and ka assumptions as well as the idea from (Halpern and Rêgo, 2009a, 2013) of different languages associated with different states.

Of related work, both Modica and Rustichini (1999) and Halpern (2001) develop logics of unawareness, but for the single-agent case only. Board and Chung (2006) add awareness operators to first order logic. Sillari (2006) also combines first-order logic and awareness, this time interpreted over neighborhood structures. There is a fundamental difference, however, between quantification in these two lat- 
ter frameworks and in that of Halpern and Rêgo (2006, 2009b,a, 2013). In (Board and Chung, 2006) and (Sillari, 2006), quantification is over objects of the universe of discourse, while in Halpern and Rêgo's work quantification is over formulae. In general, we need the latter type of quantification to reason about unawareness of formulae. Heifetz et al. (2007) develop a set theoretic framework, as opposed to the syntactic approach of Halpern and Rêgo. Our work also belongs to the syntactic tradition.

This paper is organised as follows. In the next section we introduce the logic of general awareness, and different versions of Halpern's and Rêgo's logics. Our logic of full and relative awareness is then presented in Section 3, and an axiomatisation proved sound and (weakly) complete in Section 4 . The satisfiability problem for the logic is studied in Section 5. We prove that the problem is decidable, and that it is PSPACE-complete. In Section 6 we compare the logic to Halpern's and Rêgo's logic. We conclude in Section 7.

\section{Background: Logics of Awareness and Unawareness}

In this paper we consider several logical languages $\mathscr{L}$. We define the meaning of each of these by defining the concept of a formula $\phi \in \mathscr{L}$ being true (or satisfied) in the context of the combination of a model $M \in \mathscr{M}$ in some class of models $\mathscr{M}$ and a state $s$ of $M$, written $(M, s) \models \phi$. $\phi$ is valid (with respect to $\mathscr{M}$ ), written $\models \phi$, if $(M, s) \models \phi$ for all $M \in \mathscr{M}$ and all states $s$ in $M$. We also consider (Hilbert style) logical systems $S$ over $\mathscr{L} ; \vdash_{S} \phi$ means that $\phi$ is derivable in $S$. $S$ is sound with respect to $\mathscr{M}$ iff $\vdash_{S} \phi$ implies that $=\phi$; $S$ is (weakly) complete if the converse holds. Strong completeness means that if $\Gamma \models \phi$ then $\Gamma \vdash_{S} \phi$ where $\Gamma$ may be an infinite set of formulas.

\subsection{Awareness Structures and The Logic of General Awareness}

We briefly recall the logic of general awareness (Fagin and Halpern, 1988) (our notation is similar to that of Halpern and Rêgo (2013)).

An awareness structure for $n$ agents $\{1, \ldots, n\}$ over primitive propositions $\Phi$ and logical language $\mathscr{L}$ is a tuple $\left(S, \pi, \mathscr{K}_{1}, \ldots, \mathscr{K}_{n}, \mathscr{A}_{1}, \ldots, \mathscr{A}_{n}\right)$, where $S$ is a nonempty set of states, $\pi: S \rightarrow \Phi$ says which primitive propositions are true in each state, $\mathscr{K}_{i} \subseteq S \times S$ is the accessibility relation for agent $i$, and $\mathscr{A}_{i}: S \rightarrow 2^{\mathscr{L}}$ defines the awareness set $\mathscr{A}_{i}(s) \subseteq \mathscr{L}$ for each agent $i$ in each state $s \in S$. Intuitively, $(s, t) \in \mathscr{K}_{i}$ means that when the state of the world actually is $s$ agent $i$ considers it possible that the state of the world is $t ; \phi \in \mathscr{A}_{i}(s)$ means that agent $i$ is aware of the formula $\phi$ when the state of the world is $s$.

We shall consider several model classes, defined by requiring the accessibility relations to be reflexive $\left((s, s) \in \mathscr{K}_{i}\right.$ for all $\left.s \in S\right)$, transitive $\left((s, t) \in \mathscr{K}_{i}\right.$ and $(t, u) \in$ 
$\mathscr{K}_{i}$ implies that $\left.(s, u) \in \mathscr{K}_{i}\right)$ and/or Euclidean $\left((s, t) \in \mathscr{K}_{i}\right.$ and $(s, u) \in \mathscr{K}_{i}$ implies that $\left.(t, u) \in \mathscr{K}_{i}\right)$. For $Z \subseteq\{r, t, e\}$, we use $\mathscr{M}_{n}^{Z}(\Phi, \mathscr{L})$ to denote the awareness structures for $n$ agents over $\Phi$ and $\mathscr{L}$ where the accessibility relations are required to have the properties in $Z$ ("r" means reflexive, etc.). We sometimes write $\mathscr{M}_{n}(\Phi, \mathscr{L})$ for $\mathscr{M}_{n}^{\emptyset}(\Phi, \mathscr{L})$ - the class of all awareness structures.

Given a number $n$ of agents and a set $\Phi$ of primitive propositions, the formulae $\phi$ of the language $\mathscr{L}_{n}^{K, X, A}(\Phi)$ are defined by the following grammar:

$$
\phi::=p\left|\phi_{1} \wedge \phi_{2}\right| \neg \phi\left|K_{i} \phi\right| X_{i} \phi \mid A_{i} \phi
$$

where $p \in \Phi$ and $1 \leq i \leq n$. The usual derived propositional connectives are used, for example we write $\phi \vee \psi$ for $\neg(\neg \phi \wedge \neg \psi)$ and so on. The formula $A_{i} \phi$ means that agent $i$ is aware of $\phi$.

Below we describe how awareness structures for $n$ agents over primitive propositions $\Phi$ and logical language $\mathscr{L}_{n}^{K, X, A}(\Phi)$ are used to interpret the language $\mathscr{L}_{n}^{K, X, A}(\Phi)$. In the following sections of the paper we shall also look at other languages $\mathscr{L}$, and we will then use awareness structures for $n$ agents over $\Phi$ and $\mathscr{L}$ to interpret $\mathscr{L}$.

The notion of a formula $\phi \in \mathscr{L}_{n}^{K, X, A}(\Phi)$ being true, or satisfied, in a state $s$ of an awareness structure $M=\left(S, \pi, \mathscr{K}_{1}, \ldots, \mathscr{K}_{n}, \mathscr{A}_{1}, \ldots, \mathscr{A}_{n}\right) \in \mathscr{M}_{n}\left(\Phi, \mathscr{L}_{n}^{K, X, A}(\Phi)\right)$, written $(M, s) \models \phi$, is defined as follows, where $p \in \Phi$ and $1 \leq i \leq n$ :

$$
\begin{array}{ll}
(M, s) \models p & \Leftrightarrow p \in \pi(s) \\
(M, s) \models \phi_{1} \wedge \phi_{2} & \Leftrightarrow(M, s) \models \phi_{1} \text { and }(M, s) \models \phi_{2} \\
(M, s) \models \neg \phi & \Leftrightarrow(M, s) \mid \models \phi \\
(M, s) \models K_{i} \phi & \Leftrightarrow \forall(s, t) \in \mathscr{K}_{i},(M, t) \models \phi \\
(M, s) \models A_{i} \phi & \Leftrightarrow \phi \in \mathscr{A}_{i}(s) \\
(M, s) \models X_{i} \phi & \Leftrightarrow(M, s) \models K_{i} \phi \wedge A_{i} \phi
\end{array}
$$

Example 2 (Example 1 continued). (Adapted from Halpern and Rêgo (2006)). The situation described in Example 1 up until immediately before the last sentence ("On the other hand..") can be modelled by an awareness structure $M_{2}=\left(S, \pi, \mathscr{K}_{1}, \mathscr{K}_{2}\right.$, $\left.\mathscr{A}_{1}, \mathscr{A}_{2}\right)$ for 2 agents over the set $\{p, q\}$ of primitive propositions and logical language $\mathscr{L}_{2}^{K, X, A}(\{p, q\})$, defined as follows. $S=\{s\} ; \pi(s)=\{p, q\} ; \mathscr{K}_{1}=\mathscr{K}_{2}=$ $\{(s, s)\} ; \mathscr{A}_{1}(s)=\{p\} ; \mathscr{A}_{2}(s)=\left\{p, q, A_{2} q, \neg A_{1} q, A_{2} q \wedge \neg A_{1} q\right\}$. The following hold:

- $\left(M_{2}, s\right) \models X_{1} p \wedge X_{2} p$ : both the investor and the broker explicitly know that the NASDAQ index is more likely to increase than to decrease tomorrow

- $\left(M_{2}, s\right) \models \neg X_{1} q \wedge X_{2} q$ : the investor does not explicitly know that Amazon will announce a huge increase in earnings tomorrow, but the broker does

- $\left(M_{2}, s\right) \models X_{2}\left(A_{2} q \wedge \neg A_{1} q\right)$ : the broker explicitly knows that he (broker) is aware of this fact (regarding Amazon) and the investor is not. 


\subsection{A Logic of Knowledge of Unawareness}

Halpern and Rêgo (2006) extended the logic of general awareness in order to be able to reason about knowledge of unawareness. We will refer to this logic as HR06. In describing the logic we follow Halpern and Rêgo (2013).

Let $\mathscr{X}$ be a countably infinite set of variables. The language extends the language of the logic of general awareness with variables, and formulae of the form $\forall x \phi$, where $x$ is a variable. Formulas of $\mathscr{L}_{n}^{\forall, K, X, A}(\Phi, \mathscr{X})$ are defined by the following grammar:

$$
\phi::=p\left|\phi_{1} \wedge \phi_{2}\right| \neg \phi\left|K_{i} \phi\right| X_{i} \phi\left|A_{i} \phi\right| \forall x \phi \mid x
$$

where $p \in \Phi, 1 \leq i \leq n$ and $x \in \mathscr{X}$. We use the usual abbreviations in addition to $\exists x \phi$ for $\neg \forall x \neg \phi$. A sentence is a formula without free variables; $\mathscr{S}_{n}^{\forall, K, X, A}(\Phi, \mathscr{X})$ denotes the set of all sentences.

Satisfaction of a $\mathscr{L}_{n}^{\forall, K, X, A}(\Phi, \mathscr{X})$ sentence $\phi$ is defined in relation to a pair consisting of an awareness structure $M \in \mathscr{M}_{n}\left(\Phi, \mathscr{S}_{n}^{\forall, K, X, A}(\Phi, \mathscr{X})\right)$ and a state $s$ in $M$. The domain of quantification only contains sentences of $\mathscr{L}_{n}^{K, X, A}$ (the quantifier-free language). The definition of satisfaction is by nested induction, first over the total number of free and bound variables and then on the length of the formula. The additional clause for the quantified formulas is

$$
(M, s) \models \forall x \phi \Leftrightarrow(M, s) \models \phi[x / \psi], \text { for all } \psi \in \mathscr{L}_{n}^{K, X, A}(\Phi, \mathscr{X})
$$

Example 3 (Example 2 continued). (Adapted from Halpern and Rêgo (2006)). Now we can take the last sentence in Example 1 into account in our model of the situation. Let $M_{3} \in \mathscr{M}_{n}\left(\Phi, \mathscr{S}_{n}^{\forall, K, X, A}(\Phi, \mathscr{X})\right)$ be as $M_{2}$ except that we let the investor be aware of the fact that there is something the broker is aware of but the investor is not: $\mathscr{A}_{1}(s)=\left\{p, \exists x\left(A_{2} x \wedge \neg A_{1} x\right)\right\}$.

The formulae in Example 2 continue to hold in $M_{3}$ as well. The following two formulae (from Halpern and Rêgo (2006)) illustrate reasoning about unawareness. We have that:

- $\left(M_{3}, s\right) \models X_{1}\left(\exists x\left(A_{2} x \wedge \neg A_{1} x\right)\right)$ : the investor explicitly knows that there is something that the broker is aware of but he is not

- $\left(M_{3}, s\right) \models \neg X_{2}\left(\exists x\left(A_{2} x \wedge \neg A_{1} x\right)\right)$ : the broker does not explicitly know that there is something he is aware of but the investor is not

Let $\mathbf{K}_{n, \forall}$ be the axiom system over the language $\mathscr{L}_{n}^{\forall, K, X, A}(\Phi, \mathscr{X})$ consisting of the following axioms and rules:

Prop all propositional tautologies

$\mathrm{K} \quad K_{i}(\phi \rightarrow \psi) \rightarrow\left(K_{i} \phi \rightarrow K_{i} \psi\right)$

A0 $\quad X_{i} \phi \leftrightarrow K_{i} \phi \wedge A_{i} \phi$

$1_{\forall} \forall x \phi \rightarrow \phi[x / \psi]$ if $\psi$ is quantifier free and substitutable ${ }^{2}$ for $x$ in $\phi$

\footnotetext{
${ }^{2}$ Substitutable means that no free variable of $\psi$ becomes bound as a result of the substitution.
} 


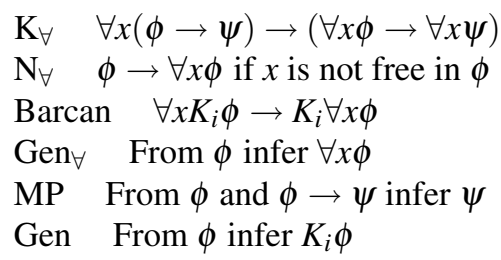

Furthermore, given the following three extra axioms,

$$
\begin{array}{ll}
\mathrm{T} & K_{i} \phi \rightarrow \phi \\
4 & K_{i} \phi \rightarrow K_{i} K_{i} \phi \\
5 & \neg K_{i} \phi \rightarrow K_{i} \neg K_{i} \phi
\end{array}
$$

$\mathbf{K}_{n, \forall}^{\mathscr{Z}}$ is the system obtained by adding axioms $\mathscr{Z}$ to $\mathbf{K}_{n, \forall}$, where $\mathscr{Z} \subseteq\{T, 4,5\}$. It is well known that $T, 4$ and 5 correspond to the accessibility relations being reflexive, transitive and Euclidean, respectively.

Theorem 1 (Halpern and Rêgo (2006)). Let $\mathscr{Z} \subseteq\{T, 4,5\}$ and let $Z$ be the corresponding subset of $\{r, t, e\}$. If $\Phi$ is countably infinite, $\mathbf{K}_{n, \forall}^{\mathscr{Z}}$ is a sound and complete axiomatisation of the language $\mathscr{L}_{n}^{\forall, K, X, A}(\Phi, \mathscr{X})$ with respect to the class of awareness structures $\mathscr{M}_{n}^{Z}\left(\Phi, \mathscr{L}_{n}^{\forall, K, X, A}(\Phi, \mathscr{X})\right)$.

Consider the addition to HR06 of the following two natural properties:

agpp: For all agents $i, \phi \in \mathscr{A}_{i}(s)$ iff all the primitive propositions that appear in $\phi$ are in $\mathscr{A}_{i} \cap \Phi$

$k a$ : For all agents $i$ and states $s, t$ such that $(s, t) \in \mathscr{K}_{i}, \mathscr{A}_{i}(s)=\mathscr{A}_{i}(t)$.

Note that the agpp implies a somewhat counterintuitive property of awareness, as also pointed out in Halpern and Rêgo (2009a). The agent is always aware of all formulas that do not contain any primitive propositions (such as $\forall x A_{i} x$ ). From the point of view of awareness common in computer science literature (an agent is aware of sentences that are represented its finite working memory or can be obtained from them using some algorithm), agpp itself is a rather counterintuitive property, since it implies that an agent is always aware of infinitely many arbitrarily complex sentences. However this property is accepted in economics literature, as is the more appealing ka property. Given these two properties, the HR06 logic derives $X_{i} \forall x A_{i} x \vee$ $X_{i} \neg \forall x A_{i} x$. In other words, the agent cannot be uncertain whether it is aware of all formulas. This is because in all $\mathscr{K}_{i}$-accessible states $s, \mathscr{A}_{i}(s)$ is the same, hence in all such $s$ the agent is either aware of all formulas or in all of them he is not aware of all formulas. However it must be possible to model an agent that is uncertain of whether he is aware of everything. This problem was addressed in (Halpern and Rêgo, 2009a, 2013). 


\subsection{A Revisited Logic of Knowledge of Unawareness}

We give a brief review of the new version of the logic as presented in (Halpern and Rêgo, 2013), henceforth called the HR13 logic. The syntax of the logic is the same as for HR06.

In order to overcome the problem with the HR06 logic mentioned in the previous section, the notion of an awareness structure is extended to include a function $\mathscr{P} \mathscr{L}$ that assigns to each state a language (a set of propositional variables). The resulting structures of the form $\left(S, \pi, \mathscr{P} \mathscr{L}, \mathscr{K}_{1}, \ldots, \mathscr{K}_{n}, \mathscr{A}_{1}, \ldots, \mathscr{A}_{n}\right)$ are referred to as extended awareness structures. In extended awareness structures over a logical language $\mathscr{L}$, it is required that every formula in $\mathscr{A}_{i}(s)$ can only contain propositional variables from $\mathscr{P} \mathscr{L}(s)$. agpp and $k a$ are assumed to hold in extended awareness structures. The class of all extended awareness structures over $\Phi$ and logical language $\mathscr{L}$ is denoted $\mathscr{N}_{n}^{Z}(\Phi, \mathscr{L})$ (where $Z$ means the same as for standard awareness structures).

The HR13 logic is interpreted in extended awareness structures over the language $\mathscr{L}_{n}^{\forall, K, X, A}(\mathscr{P} \mathscr{L}(s), \mathscr{X})$.

A formula (including negated formulae) can only be true in a state if it belongs to the language of that state. If $\phi \notin \mathscr{L}_{n}^{\forall, K, X, A}(\mathscr{P} \mathscr{L}(s), \mathscr{X})$, then both $\phi$ and $\neg \phi$ are false in $s$. For example,

$$
\begin{aligned}
& (M, s) \models p \quad \Leftrightarrow p \in \mathscr{P} \mathscr{L}(s) \text { and } p \in \pi(s) \\
& (M, s) \models \neg \phi \quad \Leftrightarrow \phi \in \mathscr{L}_{n}^{\forall, K, X, A}(\mathscr{P} \mathscr{L}(s), \mathscr{X}) \text { and }(M, s) \not \models \phi \\
& (M, s) \models \forall x \phi \Leftrightarrow(M, s) \models \phi[x / \psi], \text { for all } \psi \in \mathscr{L}_{n}^{K, X, A}(\mathscr{P} \mathscr{L}(s), \mathscr{X})
\end{aligned}
$$

Let us denote by $\mathscr{A}_{i}^{p}(s)$ the set $\mathscr{A}_{i}(s) \cap \Phi$. Clearly, in extended awareness structures, it is required that $\mathscr{A}_{i}^{p}(s) \subseteq \mathscr{P} \mathscr{L}(s)$. Given agpp, the truth definition for $A_{i} \phi$ can be equivalently rewritten as

$$
(M, s) \models A_{i} \phi \Leftrightarrow \Phi(\phi) \subseteq \mathscr{A}_{i}^{p}(s)
$$

In what follows, we will (equivalently) specify extended awareness structures using propositional awareness sets rather than awareness sets, that is, as structures of the form $\left(S, \pi, \mathscr{P} \mathscr{L}, \mathscr{K}_{1}, \ldots, \mathscr{K}_{n}, \mathscr{A}_{1}^{p}, \ldots, \mathscr{A}_{n}^{p}\right)$.

Validity is defined as follows: $\phi$ is valid in a class $\mathscr{N}$ of extended awareness structures if for all extended awareness structures $M \in \mathscr{N}$ and states $s$ such that $\Phi(\phi) \in \mathscr{P} \mathscr{L}(s),(M, s) \models \phi$.

In (Halpern and Rêgo, 2013), a new system $A X_{e}^{K, X, A, A^{*}, \forall}$ is introduced for extended awareness structures. The authors only give a soundness and completeness result for S5 (reflexive, transitive and Euclidean knowledge accessibility relations). The new system is $\mathbf{K}_{n, \forall}$ with Gen and Barcan replaced by Gen* and Barcan* and with AGPP, KA, NKA, AGPP*, A0* and FA* added. Below, $A_{i}^{*} \phi$ stands for $K_{i}(\phi \vee \neg \phi)$.

Prop all propositional tautologies 
AGPP $\quad A_{i} \phi \leftrightarrow \bigwedge_{p \in \Phi(\phi)} A_{i} p$ where $\Phi(\phi)$ is the set of primitive proposition in $\phi$

KA $\quad A_{i} \phi \rightarrow K_{i} A_{i} \phi$

NKA $\quad \neg A_{i} \phi \rightarrow K_{i} \neg A_{i} \phi$

$\mathrm{AGPP}^{*} \quad A_{i}^{*} \phi \leftrightarrow \bigwedge_{p \in \Phi(\phi)} A_{i}^{*} p$

FA* $\quad \forall x \neg A_{i}^{*} x \rightarrow K_{i} \forall x \neg A_{i}^{*} x$

$\mathrm{K} \quad K_{i}(\phi \rightarrow \psi) \rightarrow\left(K_{i} \phi \rightarrow K_{i} \psi\right)$

A0 $\quad X_{i} \phi \leftrightarrow K_{i} \phi \wedge A_{i} \phi$

$\mathrm{A} 0^{*} \quad K_{i} \phi \rightarrow A_{i}^{*} \phi$

$1_{\forall} \quad \forall x \phi \rightarrow \phi[x / \psi]$ if $\psi$ is quantifier free and substitutable ${ }^{3}$ for $x$ in $\phi$

$\mathrm{K}_{\forall} \quad \forall x(\phi \rightarrow \psi) \rightarrow(\forall x \phi \rightarrow \forall x \psi)$

$\mathrm{N}_{\forall} \quad \phi \rightarrow \forall x \phi$ if $x$ is not free in $\phi$

Barcan* $^{*}\left(A_{i}^{*}(\forall x \phi) \wedge \forall x\left(A_{i}^{*} x \rightarrow K_{i} \phi\right) \rightarrow K_{i}\left(\forall x A_{i}^{*} x \rightarrow \forall x \phi\right)\right.$

$\mathrm{Gen}_{\forall} \quad$ From $\phi$ infer $\forall x \phi$

MP From $\phi$ and $\phi \rightarrow \psi$ infer $\psi$

Gen* From $\phi$ infer $A_{i}^{*} \phi \rightarrow K_{i} \phi$

In addition,

$5^{*} \quad\left(\neg K_{i} \phi \wedge A_{i}^{*} \phi\right) \rightarrow K_{i} \neg K_{i} \phi$

Theorem 2 (Halpern and Rêgo (2013)). $A X_{e}^{K, X, A, A^{*}, \forall} \cup\left\{T, 4,5^{*}\right\}$ is a sound and complete axiomatisation of the language $\mathscr{L}_{n}^{\forall, K, X, A}(\Phi, \mathscr{X})$ with respect to $\mathscr{N}^{r, e, t}$ $\left(\Phi, \mathscr{L}_{n}^{\forall, K, X, A}(\Phi, \mathscr{X})\right)$.

It was proved in (Halpern and Rêgo, 2006, 2009b) that the HR06 logic is undecidable. There is no corresponding result for the HR13 logic, but it is arguably not the most elegant of logical systems. The purpose of the formalism proposed by Halpern and Rêgo (2013) was ease of comparison with the systems proposed in economics literature. In the next section we propose a system which can express similar properties of awareness and unawareness but is more computationally tractable and simple.

\section{A Logic of Full and Relative Awareness}

In this section we introduce the logic of full and relative awareness. It is motivated by the motivating examples of Halpern and Rêgo (2006, 2009b), but does not have variables or explicit quantification, and, furthermore, it is decidable. It also takes into account key two ideas in (Halpern and Rêgo, 2013), namely the agpp and ka properties, and the use of extended awareness structures to model different languages in different states. As discussed above, this combination makes it consistent that an agent is uncertain about being aware of everything. However, it does not incorporate the idea from (Halpern and Rêgo, 2013) of reativising truth of all formulae to the language in the current state (we view this as an orthogonal feature and

\footnotetext{
${ }^{3}$ Substitutable means that no free variable of $\psi$ becomes bound as a result of the substitution.
} 
choose a simpler framework in order to highlight the main ideas of full and relative awareness).

The language $\mathscr{L}_{n}^{C, R, K, X, A}(\Phi)$ is defined by the following grammar:

$$
\phi::=p\left|\phi_{1} \wedge \phi_{2}\right| \neg \phi\left|K_{i} \phi\right| X_{i} \phi\left|A_{i} \phi\right| C_{i} \mid R_{i j}
$$

where $p \in \Phi$ and $i, j \in[1, n]$. Note that the two new connectives $C_{i}$ and $R_{i j}$ are nullary (they don't take any arguments). $C_{i}$ is intended to mean that agent $i$ has full awareness. $R_{i j}$ is intended to mean that agent $j$ has relative awareness with respect to agent $i$, i.e., that $j$ is aware of everything $i$ is aware of.

Recall that agpp and $k a$ are assumed to hold in all extended awareness structures. Satisfaction of $\mathscr{L}_{n}^{C, R, K, X, A}(\Phi)$ formulae is defined in relation to an extended awareness structure $M \in \mathscr{N}_{n}\left(\Phi, \mathscr{L}_{n}^{C, R, K, X, A}(\Phi)\right)$ and a state $s$ of $M$.

$$
\begin{array}{ll}
(M, s) \models p & \Leftrightarrow p \in \pi(s) \\
(M, s) \models \phi_{1} \wedge \phi_{2} & \Leftrightarrow(M, s) \models \phi_{1} \text { and }(M, s) \models \phi_{2} \\
(M, s) \models \neg \phi & \Leftrightarrow(M, s) \not \models \phi \\
(M, s) \models K_{i} \phi & \Leftrightarrow \forall(s, t) \in \mathscr{K}_{i},(M, t) \models \phi \\
(M, s) \models A_{i} \phi & \Leftrightarrow \Phi(\phi) \subseteq \mathscr{A}_{i}^{p}(s) \\
(M, s) \models X_{i} \phi & \Leftrightarrow(M, s) \models K_{i} \phi \wedge A_{i} \phi \\
(M, s) \models C_{i} & \Leftrightarrow \mathscr{A}_{i}^{p}(s)=\mathscr{P}_{\mathscr{L}}(s) \\
(M, s) \models R_{i j} & \Leftrightarrow \mathscr{A}_{i}^{p}(s) \subseteq \mathscr{A}_{j}^{p}(s)
\end{array}
$$

Note that unlike HR13 we do not require that the formula belongs to the language of $s$ in order for it to be true in $s$. We essentially interpret $\mathscr{P} \mathscr{L}(s)$ as the set of primitive propositions that agents can be in principle aware of in $s$, which is different from the (larger) set of propositions which may be true or false in $s$. We adopt this change since it simplifies the technical developments, but also because we find the distinction between the propositions that agents may be aware of (given their subjective limitations) in a given state, and objective properties that may be true or false in all states intuitively acceptable. For example, in the Middle Ages people could not be possibly aware of $p$ where $p$ is a statement that the hydrogen atom consists of one proton and one electron, however one could argue that $p$ was still true.

Note that $C_{i}$ cannot be expressed by a finite conjunction of the form $A_{i} p_{1} \wedge A_{i} p_{2}$ $\wedge A_{i} p_{3} \wedge \ldots$ since $\mathscr{P} \mathscr{L}(s)$ is different in different $s$ and also because it may be infinite. $\neg C_{i}$ means that there exists a primitive proposition $p$ such that $p \notin \mathscr{A}_{i}^{p}(s)$. Thus, $X_{i} \neg C_{i}$ expresses knowledge of unawareness: agent $i$ explicitly knows that there is something he is unaware of. $R_{i j}$ means that $i$ 's awareness set is included in $j$ 's awareness set, that $j$ is aware of everything $i$ is aware of. $\neg R_{i j}$ means that there is something $i$ is aware of but $j$ is not.

It is possible that $K_{i} \neg C_{i}$ is true, without there being any $\phi$ such that $K_{i} \neg A_{i} \phi$ is true, and it is possible that $K_{i} \neg R_{j i}$ is true without there being any $\phi$ such that $K_{i}\left(A_{j} \phi \wedge \neg A_{i} \phi\right)$ is true. 
Example 4 (Example 3 continued). Let $M_{4}$ be an extended awareness structure with the same components and the same propositional awareness sets as $M_{3}$ from 3 . The fact that there is something that the broker is aware of but the investor is not aware of can now be expressed by the formula $\neg R_{21}$.

The formula can now be expressed as follows:

- $\left(M_{4}, s\right) \models X_{1}\left(\neg R_{21}\right)$

Note that the logic is not compact. As a counter example take the theory $\left\{\neg C_{i}\right\} \cup\left\{A_{i} \phi: \phi \in \mathscr{L}_{n}^{C, R, K, X, A}(\Phi)\right\}$, or the theory $\left\{\neg R_{i j}\right\} \cup\left\{\neg A_{i} \phi \vee A_{j} \phi: \phi \in\right.$ $\left.\mathscr{L}_{n}^{C, R, K, X, A}(\Phi)\right\}$.

In the next section, we present an axiomatisation of the logic.

\section{Axiomatisation}

Let $\mathscr{S}$ be the axiom system consisting of the following axioms and inference rules, over the language $\mathscr{L}_{n}^{C, R, K, X, A}(\Phi)$ :

Prop all propositional tautologies

$\mathrm{K} \quad K_{i}(\phi \rightarrow \psi) \rightarrow\left(K_{i} \phi \rightarrow K_{i} \psi\right)$

AGPP $\quad A_{i} \phi \leftrightarrow \bigwedge_{p \in \Phi(\phi)} A_{i} p$

KA $\quad A_{i} \phi \rightarrow K_{i} A_{i} \phi$

NKA $\quad \neg A_{i} \phi \rightarrow K_{i} \neg A_{i} \phi$

A0 $\quad X_{i} \phi \leftrightarrow K_{i} \phi \wedge A_{i} \phi$

A1 $\quad R_{i j} \rightarrow\left(A_{i} \phi \rightarrow A_{j} \phi\right)$

A2 $R_{i i}$

A3 $\quad R_{i j} \wedge R_{j k} \rightarrow R_{i k}$

C1 $\quad C_{i} \rightarrow A_{i} \phi$

$\mathrm{C} 2 \quad C_{i} \rightarrow R_{j i}$

$\mathrm{C} 3 \quad\left(C_{i} \wedge R_{i j}\right) \rightarrow C_{j}$

MP From $\phi$ and $\phi \rightarrow \psi$ infer $\psi$

Gen From $\phi$ infer $K_{i} \phi$

Prop, K, A0, MP and Gen axiomatise the logic of general awareness (Fagin and Halpern, 1988). AGPP, KA and NKA correspond to agpp (awareness generated by primitive propositions) and $k a$ (the agents know what they are and are not aware of), respectively. ${ }^{4}$ A1 says that relative awareness implies that the agent with greater

${ }^{4}$ The system $\mathscr{S}$ without AGPP, KA and NKA has been shown in Ågotnes and Alechina (2007) to be sound and complete for the class of awareness structures (without the assumption of different language in different states, agpp and $\mathrm{ka}$ ) with the truth definitions for $C_{i}$ and $R_{i j}$ stated as follows:

$$
\begin{aligned}
& (M, s) \models C_{i} \Leftrightarrow \mathscr{A}_{i}(s)=\mathscr{L}_{n}^{C, R, K, X, A}(\Phi) \\
& (M, s) \models R_{i j} \Leftrightarrow \mathscr{A}_{i}(s) \subseteq \mathscr{A}_{j}(s)
\end{aligned}
$$


awarenesss is aware of any formula the other agent is aware of. A2 and A3 say that relative awareness is reflexive and transitive, respectively. $\mathrm{C} 1$ says that full awareness implies awareness of any particular formula. C2 says that full awareness implies relative awareness (with respect to any other agent), and C3 says that relative awareness implies full awareness in the case that the other agent has full awareness.

Furthermore, $\mathscr{S}^{\mathscr{Z}}$ is the system obtained by adding axioms $\mathscr{Z}$ to $\mathscr{S}$, where $\mathscr{Z} \subseteq\{T, 4,5\}$.

The following theorem shows that the axiomatisation is sound and weakly complete $^{5}$.

Theorem 3 (Soundness and Weak Completeness). Let $\mathscr{Z} \subseteq\{T, 4,5\}$ and let $Z$ be the corresponding subset of $\{r, t, e\} . \mathscr{S}^{\mathscr{Z}}$ is a sound and weakly complete axiomatisation of the language $\mathscr{L}_{n}^{C, R, K, X, A}(\Phi)$ with respect to $\mathscr{N}_{n}^{Z}\left(\Phi, \mathscr{L}_{n}^{C, R, K, X, A}(\Phi)\right)$.

Proof. Soundness is straightforward.

For completeness, let $\phi$ be a $\mathscr{S}^{\mathscr{Z}}$ consistent formula. We will show that $\phi$ is satisfiable in $\mathscr{N}_{n}^{Z}\left(\Phi, \mathscr{L}_{n}^{C, R, K, X, A}(\Phi)\right)$, which completes the proof.

First, we build a canonical (standard) Kripke structure $M^{c}=\left(S^{c}, \pi, \mathscr{K}_{1}, \ldots, \mathscr{K}_{n}\right)$ in the standard way:

- $S^{c}$ is the set of all maximal $\mathscr{S}^{\mathscr{Z}}$ consistent sets of formulae

- $(s, t) \in \mathscr{K}_{i}$ iff $K_{i} \psi \in s$ implies that $\psi \in t$, for all formulae $\psi$

- $p \in \pi(s)$ iff $p \in s$

Note that $M^{c}$ is guaranteed to satisfy the required properties of $\mathscr{K}_{i}$. If $r \in Z$, $T \in \mathscr{Z}$ ensures that each $\mathscr{K}_{i}$ is reflexive, and similarly for $t / 4$ and $e / 5$ (can be shown in the standard way).

We are going to construct an extended awareness structure $M=\left(S^{c}, \pi, \mathscr{P} \mathscr{L}, \mathscr{K}_{1}\right.$, $\left.\ldots, \mathscr{K}_{n}, \mathscr{A}_{1}, \ldots, \mathscr{A}_{n}\right)$ that satisfies agpp, ka and the Truth Lemma in three stages. By the Truth Lemma, we mean the following property. Let $\operatorname{Subf}(\phi)$ be the set of subformulas of $\phi$ closed under single negation. The Truth Lemma is as follows: for every formula $\psi \in \operatorname{Subf}(\phi)$,

$$
(M, s) \models \psi \Leftrightarrow \psi \in s
$$

In the first stage, we will construct $M_{1}$ that satisfies agpp, $k a$ and the truth lemma for propositional formulas, formulas of the form $A_{i} \psi, K_{i} \psi$ and $X_{i} \psi$ (but not $R_{i j}$ and $C_{i}$ ). Then we will construct $M_{2}$ where in addition the truth lemma holds for all subformulas of $\phi$, but $k a$ does not. Then finally we construct $M$ by enforcing $k a$ while preserving agpp and the truth lemma for all types of subformulas of $\phi$.

To construct $M_{1}$, we add to $M^{c}$ awareness sets constructed in a straightforward way:

- $\psi \in \mathscr{A}_{i}(s)$ iff $A_{i} \psi \in \operatorname{Subf}(\phi)$ and $A_{i} \psi \in s$.

\footnotetext{
${ }^{5}$ Note that weak completeness of our logic does not imply strong completeness, because the logic is not compact: it is possible to exhibit an infinite unsatisfiable set of formulas every finite subset of which is satisfiable.
} 
Note that the AGPP axiom guarantees that this is equivalent to the following condition:

- $p \in \mathscr{A}_{i}^{p}(s)$ iff for some $A_{i} \psi \in s, A_{i} \psi \in \operatorname{Subf}(\phi)$ and $p \in \Phi(\psi)$

In what follows, we will be working with $\mathscr{A}_{i}^{p}(s)$ sets rather than with $\mathscr{A}_{i}(s)$. We also set

- $\mathscr{P} \mathscr{L}(s)=\cup_{i} \mathscr{A}_{i}^{p}(s)$

It is easy to check that $M_{1}$ satisfies agpp, $k a$ (because of KA and NKA axioms) and the truth lemma for boolean formulas, formulas of the form $A_{i} \psi, K_{i} \psi$ and $X_{i} \psi$. However, the truth lemma does not hold for subformulas of $\phi$ of the form $R_{i j}$ and $C_{i}$. For example, it is possible that $\neg R_{i j} \in s$ but $\mathscr{A}_{i}^{p}(s)=\mathscr{A}_{j}^{p}(s)$, or that $\neg C_{i} \in s$ but $\mathscr{A}_{i}^{p}(s)=\mathscr{P} \mathscr{L}(s)$. Next, we construct $M_{2}$ where we add extra propositional variables to propositional awareness sets and fix this problem 'locally' in each state, so that the truth lemma holds for each $s$. However $k a$ does not hold any longer because the fixes are different in different states.

The construction of $M_{2}$ only involves changes to the propositional awareness sets $\mathscr{A}_{i}^{p}$ and to $\mathscr{P} \mathscr{L}$, and proceeds in $n+1$ steps. Let $q_{1}, q_{2}, \ldots, q_{n+1}$ be a set of propositional variables not occurring in $\operatorname{Subf}(\phi)$. For each agent $i$, set $X_{0}^{i}$ to be $\mathscr{A}_{i}^{p}(s)$ from $M_{1}$ and $\mathscr{P} \mathscr{L}^{0}(s)$ to $\mathscr{P} \mathscr{L}(s)$ from $M_{1}$. At the step corresponding to agent $i$, if $\neg R_{i j} \in s$ then add $q_{i}$ to $X_{k}^{i}$, for every $k$ such that $R_{i k} \in s$ (including $X_{i}^{i}$ ). We also add $q_{i}$ to $\mathscr{P} \mathscr{L}^{i}(s)$ and reassign $X_{m}^{i}(s)$ to be $\mathscr{P} \mathscr{L}^{i}(s)$ for all $m$ such that $C_{m} \in s$. Finally, we set $\mathscr{P} \mathscr{L}^{n+1}(s)$ to be $\mathscr{P} \mathscr{L}^{n}(s) \cup\left\{q_{n+1}\right\}$ and set $X_{m}^{n+1}(s)$ to be $\mathscr{P} \mathscr{L}^{n+1}(s)$ for all $m$ such that $C_{m} \in s$. (The latter step is to deal with the situation when for some $m, X_{m}^{n}(s)=\mathscr{P} \mathscr{L}^{n}(s)$ but $\neg C_{m} \in s$.) We set $\mathscr{A}_{i}^{p}(s)$ in $M_{2}$ to be $X_{i}^{n+1}$ and $\mathscr{P} \mathscr{L}(s)$ to be $\mathscr{P} \mathscr{L}^{n+1}$. It is easy to check that now the truth lemma holds for all subformulas of $\phi$. However, now $k a$ does not hold because it is possible that for some $(s, t) \in \mathscr{K}_{i}$ that $R_{i j} \in s$ and $\neg R_{i j} \in t$, so $q_{i} \in \mathscr{A}_{i}^{p}(t)$ but $q_{i} \notin \mathscr{A}_{i}^{p}(s)$.

In the last stage of the construction, we modify $M_{2}$ to obtain $M$ where $k a$ holds, as well as the other properties of extended awareness structures. In addition, we want the truth lemma for subformulas of $\phi$ to continue to hold. For the latter, it is sufficient to maintain the following four properties:

(R) if $R_{i j} \in s$ then $\mathscr{A}_{i}^{p}(s) \subseteq \mathscr{A}_{j}^{p}(s)$

(C) if $C_{i} \in s$ then $\mathscr{A}_{i}^{p}(s)=\mathscr{P} \mathscr{L}(s)$

(notR) if $\neg R_{i j} \in s$ then $\mathscr{A}_{i}^{p}(s) \not \mathscr{A}_{j}^{p}(s)$

(notC) if $\neg C_{i} \in s$ then $\mathscr{A}_{i}^{p}(s) \subset \mathscr{P} \mathscr{L}(s)$

In constructing $M$, we first unravel $M_{2}$ from some state $s_{0}$ such that $\phi \in s_{0}$ (preserving symmetry, transitivity etc. of $\mathscr{K}_{i}$ as required) so that for every $s$ and $t$ in the new structure, $(s, t) \in \mathscr{K}_{i}$ for at most one $i$ (but possibly $\left(s, t^{\prime}\right) \in \mathscr{K}_{j}$ for $t^{\prime} \neq t$ and $j \neq i$ ). This establishes the property that in $M$, it is not possible to go from $s$ to $t(s \neq t)$ by $\mathscr{K}_{i}$ and by $\mathscr{K}_{j}(j \neq i)$ (or to come back from $t$ to $s$ by $\mathscr{K}_{j}$ ). This rules out impossible to fix situations like $R_{i j} \in s,(s, t) \in \mathscr{K}_{i} \cap \mathscr{K}_{j}$, and $\neg R_{i j} \in t$ (this would mean that $\mathscr{A}_{i}^{p}(s)=\mathscr{A}_{i}^{p}(t), \mathscr{A}_{j}^{p}(s)=\mathscr{A}_{j}^{p}(t)$, but in $s$ we need to ensure that 
$\mathscr{A}_{i}^{p}(s) \subseteq \mathscr{A}_{j}^{p}(s)$ and in $\left.t, \mathscr{A}_{i}^{p}(t) \nsubseteq \mathscr{A}_{j}^{p}(t)\right)$ and enables the construction below of a model where $k a$ holds for all states and agents.

Let $K(s, i)=\left\{t:(s, t) \in T C\left(\mathscr{K}_{i}\right)\right\}$, where $T C\left(\mathscr{K}_{i}\right)$ is the reflexive transitive closure of $\mathscr{K}_{i}$. (So $s \in K(s, i)$ ). We need transitive closure because if $\mathscr{K}_{i}$ is not transitive, then after we 'fix' $\mathscr{A}_{i}^{p}(t)$ for some $t$ accessible by $\mathscr{K}_{i}$ from $s$ to ensure $\mathscr{A}_{i}^{p}(s)=$ $\mathscr{A}_{i}^{p}(t)$, we may need to 'fix' it again because $\left(t, t^{\prime}\right) \in \mathscr{K}_{i}$ and $\mathscr{A}_{i}^{p}(t) \subset \mathscr{A}_{i}^{p}\left(t^{\prime}\right)$, and this will 'undo' the fix to $k a$ for $i$ in $s$.) Observe that because of KA and NKA axioms, all states in $K(s, i)$ contains the same formulas of the form $A_{i} \psi$.

Note that for every $s, i$, and $j \neq i, K(s, i) \cap K(s, j) \subseteq\{s\}$ because of the unravelling.

Let $A(s, i)$ be $\bigcup_{t \in K(s, i)} \mathscr{A}_{i}^{p}(t) . A(s, i)$ is finite since it contains only propositional variables in $\operatorname{Subf}(\phi)$ and at most $n+1$ additional propositional variables used for 'local fixing' to compute propositional awareness sets in $M_{2}$. Also observe that although $K(s, i)$ may be infinite, it contains only finitely many 'types' of states with respect to the contents of their propositional awareness sets and the pattern of $R_{i j}$ and $C_{i}$ formulas (since the number of agents is finite). We are going to make all propositional awareness sets $\mathscr{A}_{i}^{p}(t)$ for $t \in K(s, i)$ the same by extending them to be equal to $A(s, i)$. Note that $\mathscr{A}_{i}^{p}(t)$ only differ from $A(s, i)$ in the new propositional variables used as witnesses (since they agree on all $p \in \Phi(\phi)$ ). The procedure is as follows:

For all $q \in A(s, i)$ :

For all $t \in K(s, i)$ such that $t \notin \mathscr{A}_{i}^{p}(t)$ :

step $1 \quad \mathscr{A}_{i}^{p}(t):=\mathscr{A}_{i}^{p}(t) \cup\{q\}$

step 2 for all $k$ with $R_{i k} \in t, \mathscr{A}_{k}^{p}(t):=\mathscr{A}_{k}^{p}(t) \cup\{q\}$ (note that this also adds $q$ to

$\mathscr{A}_{k}^{p}(t)$ for all $k$ such that $C_{k} \in t$, since $R_{i k} \in t$ by axiom C2.)

step 3 if $q \notin \mathscr{P} \mathscr{L}(t), \mathscr{P} \mathscr{L}(t):=\mathscr{P} \mathscr{L}(t) \cup\{q\}$

It is easy to check that this procedure terminates (if we interpret iterating over $t \in$ $K(s, i)$ as iterating over finitely many 'types' of $t)$ and results in all $\mathscr{A}_{i}^{p}(t)$ being equal. It also makes sure that the conditions (R) and (C) which are necessary for the truth lemma are satisfied. However we may have broken the conditions (notR) and (notC): it is possible that after adding an extra $q$ to some $\mathscr{A}_{k}^{p}(t)$ with $R_{i k} \in t$ we destroyed a witness for $\neg R_{j k} \in t$ for some $j$ (if $q=q_{j}$ ) or a witness for $\neg C_{k} \in t$ (if $\left.q=q_{n+1}\right)$. So we add two extra steps of 'fixing' those witnesses:

step $4 \quad$ if $\neg R_{j k} \in t$ and $\mathscr{A}_{j}^{p}(t)=\mathscr{A}_{k}^{p}(t)$, add a new $q^{\prime}$ to $\mathscr{A}_{j}^{p}(t)$ and all $\mathscr{A}_{m}^{p}(t)$ for $m$ with $R_{j m}$ and to $\mathscr{P} \mathscr{L}(t)$

step 5 if $\neg C_{k} \in t$ and $\mathscr{A}_{j}^{p}(t)=\mathscr{P} \mathscr{L}(t)$, add a new $q^{\prime \prime}$ to $\mathscr{P} \mathscr{L}(t)$

Note that steps 4 and 5 do not affect $\mathscr{A}_{i}^{p}(t)$. Let us consider step 4. Suppose $\neg R_{j k} \in t$ such that after adding some propositional variable $q$ to $\mathscr{A}_{i}^{p}(t)$ and to $\mathscr{A}_{k}^{p}(t)$ for $k$ with $R_{i k}, \mathscr{A}_{j}^{p}(t)=\mathscr{A}_{k}^{p}(t)$. We assume that before that $\mathscr{A}_{j}^{p}(t) \nsubseteq \mathscr{A}_{k}^{p}(t)$. This can only happen if previously $q \in \mathscr{A}_{j}^{p}(t)$ and $q \notin \mathscr{A}_{k}^{p}(t)$ and $q$ was added to $\mathscr{A}_{k}^{p}(t)$. In other words, $q$ was the witness for $\neg R_{j k}$. Note that this entails that $j \neq i$ (since $q$ was added in step 1 or step 2, either $k=i$ or $R_{i k} \in t$, hence if $j$ were equal to $i$ we would get a contradiction with $\neg R_{j k}$ ). This also entails that $C_{i} \notin t$ (by the same reasoning, if $i=k$ or $R_{i k} \in t$ and $\neg R_{j k} \in t$, we would get a contradiction with $R_{j i}$ that follows 
from $\left.C_{i}\right)$. So we can fix $\neg R_{j k}$ by adding a new proposition to $\mathscr{A}_{j}^{p}(s)$ and propagating it to $\mathscr{A}_{m}^{p}$ with $R_{j m}$ and the language of $t$; this will never involve changing $\mathscr{A}_{i}^{p}(t)$.

Similarly, in step 5 , if for some $k$ with $\neg C_{k} \in t$ after propagating new propositional variables from $\mathscr{A}_{i}^{p}(t)$ on step 2 causes $\mathscr{A}_{k}^{p}(t)=\mathscr{P} \mathscr{L}(t)$, this means that $q$ was the witness for $\neg C_{k}$. Again we add a new propositional variable as a witness for $\neg C_{k}$ to $\mathscr{P} \mathscr{L}(t)$ and all awareness sets $\mathscr{A}_{m}^{p}(t)$ such that $C_{m} \in t$. Note that by the previous reasoning $\neg C_{i} \in t$ and this procedure does not affect $\mathscr{A}_{i}^{p}(t)$.

Let us denote the set of new propositional variables used in steps 4 and $5 D(s, i)$. Note that $A(s, i) \cap D(s, i)=\emptyset$. We use a new propositional variable for every instance of steps 4 and 5 , so for $i \neq j, D(s, i) \cap D(s, j)=\emptyset$.

This finishes the description of how, for a single state $s$ and agent $i$, we can enforce $k a$ for $s$ and $\mathscr{K}_{i}$. To enforce $k a$ globally (for all states and all agents) we start in $s_{0}$ and fix $K\left(s_{0}, i\right)$ for each $i$. Note that the only state that the sets $K\left(s_{0}, i\right)$ for different $i$ s have in common is at most $s_{0}$ itself. However it is not sufficient to fix each $K\left(s_{0}, i\right)$ once since it is possible that $R_{i j}$ and $R_{j i}$ are in $s_{0}$ for different $i$ and $j$. This means that after $K\left(s_{0}, i\right)$ is fixed it is possible that the fix to $K\left(s_{0}, j\right)$ changes $\mathscr{A}_{i}^{p}\left(s_{0}\right)$ and it needs to be fixed again. However since the sets $D(s, i)$ and $D(s, j)$ are disjoint, no new faults which need fixed by steps 4 and 5 will occur after the second execution of steps $1-3$.

To sum up, we have constructed a model $M$ where $k a$ and agpp hold and the conditions on $\mathscr{K}_{i}$ (reflexivity, transitivity and symmetry) hold if the corresponding axioms are present in the logic. Thus, $M \in \mathscr{N}_{n}^{Z}\left(\Phi, \mathscr{L}_{n}^{C, R, K, X, A}(\Phi)\right)$. Since $\phi \in s_{0}$, the truth lemma will imply that $M, s_{0} \models \phi$ so $\phi$ is satisfiable.

The proof of the truth lemma is by structural induction over $\psi \in \operatorname{Subf}(\phi)$ :

- $\psi=A_{i} \gamma$ : for the direction to the left, $A_{i} \gamma \in s$ implies that $\Phi(\gamma) \in X_{i}^{0} \subseteq \mathscr{A}_{i}^{p}(s)$ hence $M, s \models A_{i} \gamma$. For the direction to the right, let $\Phi(\gamma) \in \mathscr{A}_{i}^{p}(s)$. If $C_{i} \in s$ then $A_{i} \gamma \in s$ by C1. If $C_{i} \notin s$, it must be the case that $\Phi(\gamma) \in X_{i}^{0}$ since $\gamma \in \operatorname{Subf}(\phi)(\gamma$ cannot contain any witness propositions added in the construction of $\left.M_{1}, M_{2}, M\right)$, and thus $\wedge_{p \in \Phi(\gamma)} A_{i} p \in s$ and by AGPP, $A_{i} \gamma \in s$.

- $\psi=R_{i j}$ : for the direction to the left, let $R_{i j} \in s$. By (R), $\mathscr{A}_{i}^{p}(s) \subseteq \mathscr{A}_{j}^{p}(s)$ hence $M, s \models R_{i j}$. For the direction to the right, let $R_{i j} \notin s$, then $\neg R_{i j} \in s$, hence by (notR), $\mathscr{A}_{i}(s) \not \mathscr{A}_{j}(s)$, so $M, s \not \models R_{i j}$ hence $M, s \models \neg R_{i j}$.

- $\psi=C_{i}$ : For the direction to the left, let $C_{i} \in s . \mathscr{A}_{i}^{p}(s)=\mathscr{P} \mathscr{L}(s)$ by (C), so $(M, s) \models C_{i}$.

For the direction to the right, let $\mathscr{A}_{i}^{p}(s)=\mathscr{P} \mathscr{L}(s)$. The only way that can happen is when $C_{i} \in s$ (otherwise, $\neg C_{i} \in s$, and by $(\operatorname{notC}), \mathscr{A}_{i}^{p}(s) \subset \mathscr{P} \mathscr{L}(s)$.

- $\psi=K_{i} \gamma$ : this case can be shown in the standard way.

Let $K_{i} \gamma \in s$. To show that $(M, s) \models K_{i} \gamma$, consider an arbitrary $t$ such that $\mathscr{K}_{i}(s, t)$. By the definition of $\mathscr{K}_{i}, \gamma \in t$, and by the inductive hypothesis $(\gamma \in \operatorname{Subf}(\phi))$ $(M, t) \mid=\gamma$. Hence, $(M, s) \models K_{i} \gamma$.

Let $K_{i} \gamma \notin s$. We will find a $t$ with $\mathscr{K}_{i}(s, t)$ such that $(M, t) \not \models \gamma$. This will show that $(M, s) \not K_{i} \gamma$. Consider the set $\{\neg \gamma\} \cup\left\{\chi: K_{i} \chi \in s\right\}$. This set is consistent (otherwise $\vdash_{\mathscr{S}} \chi_{1} \wedge \ldots \wedge \chi_{k} \rightarrow \gamma$ for some $\chi_{1}, \ldots, \chi_{k}$ from this set, hence $\vdash_{\mathscr{S}}$ 
$K_{i} \chi_{1} \wedge \ldots \wedge K_{i} \chi_{k} \rightarrow K_{i} \gamma$, which would force $\left.K_{i} \gamma \in s\right)$. So, it can be extended to a mcs $t$. Since $\neg \gamma \in t, \gamma \notin t$, and by the inductive hypothesis $(M, t) \not \models \gamma$.

- The cases for atomic propositions, $\neg$ and $\wedge$ are straightforward.

\section{Decidability and Complexity}

We are going to show that the satisfiability problem for $\mathscr{N}_{n}^{Z}\left(\Phi, \mathscr{L}_{n}^{C, R, K, X, A}(\Phi)\right)$ for any $Z \subseteq\{r, t, e\}$ is decidable in PSPACE.

Theorem 4 (Complexity). The satisfiability problem for $\mathscr{N}_{n}^{Z}\left(\Phi, \mathscr{L}_{n}^{C, R, K, X, A}(\Phi)\right)$ for any $Z \subseteq\{r, t, e\}$ is PSPACE-complete.

Proof. PSPACE-hardness follows from the results for corresponding multi-modal logics, see Halpern and Moses (1992).

To show PSPACE upper bound, we adapt the tableau algorithm of Halpern and Moses (1992) for logics $K_{n}^{Z}, Z \subseteq\{T, 4,5\}$. A tableau for $K_{n}^{Z}$ is a tuple $T=\left(S, L, \mathscr{K}_{1}, \ldots, \mathscr{K}_{n}\right)$, where $S$ is a set of states, $\mathscr{K}_{i}$ for each agent $i$ in a binary relation on $S$, and $L$ is a labelling function which associates with each state $s \in S$ a set $L(s)$ of formulas such that

PT $L(s)$ is a propositional tableau (that is, a set of formulas satisfying (PT(a)) if $\neg \neg \psi \in L(s)$ then $\psi \in L(s)$; (PT(b)) if $\psi \wedge \psi^{\prime} \in L(s)$, then $\psi, \psi^{\prime} \in L(s)$; (PT(c)) if $\neg\left(\psi \wedge \psi^{\prime}\right) \in L(s)$, then either $\neg \psi \in L(s)$ or $\neg \psi^{\prime} \in L(s)$; and (PT(d)) for no $\psi$, $\psi \in L(s)$ and $\neg \psi \in L(s)$

$\mathrm{K} 1 \quad$ if $K_{i} \psi \in L(s)$ and $(s, t) \in \mathscr{K}_{i}$, then $\psi \in L(t)$

$\mathrm{K} 2 \quad$ if $\neg K_{i} \psi \in L(s)$, then there exists $t$ with $(s, t) \in \mathscr{K}_{i}$ and $\neg \psi \in L(t)$

$\mathrm{T}$ a $T_{n}$ tableau in addition satisfies the condition if $K_{i} \psi \in L(s)$, then $\psi \in L(s)$

4 a $4_{n}$ tableau satisfies the condition if $K_{i} \psi \in L(s)$ and $(s, t) \in \mathscr{K}_{i}$, then $K_{i} \psi \in L(t)$

5 a $5_{n}$ tableau satisfies the condition if $(s, t),(s, u) \in \mathscr{K}_{i}$, and $K_{i} \psi \in L(t)$, then $K_{i} \psi, \psi \in L(u)$.

$S 5_{n}(Z=\{T, 4,5\})$ tableaux have a simpler condition, namely if $(s, t) \in \mathscr{K}_{i}$, then $K_{i} \psi \in L(s)$ iff $K_{i} \psi \in L(t)$.

A tableau $T$ is a tableau for $\phi$ if $\phi \in L(s)$ for some $s \in S$. Halpern and Moses (1992) prove that a modal formula $\phi$ is $K_{n}^{Z}$-satisfiable iff there is a $K_{n}^{Z}$ tableau for $\phi$. Together with a terminating algorithm for constructing a tableau for a given formula, this gives a decidability proof for the multi-modal logics $K_{n}^{Z}$. Showing that a tableau can be constructed using space polynomial in the size of the formula gives the PSPACE complexity result. The algorithm uses the following terminology. A set of formulas $\Gamma$ is called fully expanded if for every formula $\phi \in \Gamma$ and a subformula $\psi$ of $\phi$, either $\psi \in \Gamma$ or $\neg \psi \in \Gamma$. $\psi \in \Gamma$ is a witness that $\Gamma$ is not a propositional tableau if one of the clauses (PT(a))-(PT(c)) with $\Gamma$ in place of $L(s)$ does not apply 
to $\psi$; similarly $\psi$ is a witness that $\Gamma$ is not fully expanded if $\psi$ is a subformula of some $\phi \in \Gamma$ and neither $\psi$ nor $\neg \psi$ are in $\Gamma$. $\Gamma$ is blatantly inconsistent if (PT(d)) with $\Gamma$ in place of $L(s)$ is violated.

Below we give the algorithm for constructing a $K_{n}$ tableau for a formula $\phi_{0}$ from Halpern and Moses (1992) and modifications for $K_{n}^{Z}$, and then show how to extend the algorithm for $\mathscr{L}_{n}^{C, R, K, X, A}(\Phi)$ formulas. The algorithm below constructs a pretableau for $\phi_{0}$; a tableau is obtained by keeping only the fully expanded and not blatantly inconsistent nodes (states) and only the edges labelled by some agent $i$ between them (corresponding to $\mathscr{K}_{i}$ ).

1. Construct a tree consisting of a single root node $s_{0}$ with $L\left(s_{0}\right)=\left\{\phi_{0}\right\}$.

2. Repeat until none of (a)-(d) below applies:

a. Forming a propositional tableau: if $s$ is a leaf of the tree, $L(s)$ is not blatantly inconsistent, $L(s)$ is not a propositional tableau, and $\psi$ is the first (in some lexicographic ordering of formulas) witness to this fact, then

i. if $\psi$ is of the form $\neg \neg \psi^{\prime}$, then create a child $s^{\prime}$ of $s$ in the tree and set $L\left(s^{\prime}\right)=L(s) \cup\left\{\psi^{\prime}\right\}$

ii. if $\psi$ is of the form $\psi_{1} \wedge \psi_{2}$, then create a child $s^{\prime}$ of $s$ in the tree and set $L\left(s^{\prime}\right)=L(s) \cup\left\{\psi_{1}, \psi_{2}\right\}$

iii. if $\psi$ is of the form $\neg\left(\psi_{1} \wedge \psi_{2}\right)$, then create two children $s_{1}$ and $s_{2}$ of $s$ and set $L\left(s_{i}\right)=L(s) \cup\left\{\psi_{i}\right\}$.

b. Forming a fully expanded propositional tableau: if $s$ is a leaf of the tree, $L(s)$ is not blatantly inconsistent, $L(s)$ is not a propositional tableau, and $\psi$ is the first (in some lexicographic ordering of formulas) witness to this fact, then create two children $s_{1}$ and $s_{2}$ of $s$ and set $L\left(s_{1}\right)=L(s) \cup\{\psi\}, L\left(s_{2}\right)=L(s) \cup\{\neg \psi\}$.

c. Creating $i$-successor nodes: if $s$ is a leaf of the tree, $L(s)$ is not blatantly inconsistent, and $L(s)$ is a fully expanded propositional tableau, then for each formula of the form $\neg K_{i} \psi \in L(s)$ create an $i$-successor node $s^{\prime}$ (add an edge from $s$ to $s^{\prime}$ to the tree labelled $i$ ) and let $L\left(s^{\prime}\right)=\left\{\psi^{\prime}: K_{i} \psi^{\prime} \in L(s)\right\} \cup\{\neg \psi\}$.

d. Marking nodes satisfiable: if $s$ is not marked satisfiable then mark $s$ satisfiable if either $L(s)$ is not a fully expanded propositional tableau and some successor $s^{\prime}$ of $s$ is marked satisfiable, or $L(s)$ is a fully expanded propositional tableau, there are no formulas of the form $\neg K_{i} \psi$ in $L(s)$, and $L(s)$ is not blatantly inconsistent, or $L(s)$ is a fully expanded propositional tableau, $s$ has successors, and all of them are marked satisfiable.

3. If the root of the tree is marked satisfiable, then return ' $\phi_{0}$ is satisfiable', otherwise return ' $\phi_{0}$ is not satisfiable'.

To produce a tableau for $T_{n}$, an additional condition is added for marking nodes as satisfiable: a node is not marked as satisfiable if it contains $K_{i} \psi$ and $\neg \psi$ for some $\psi$.

Step 2(c) for $4_{n}$ and $5_{n}$ is modified slightly to ensure that the construction terminates. To be precise, step 2 (c) for $4_{n}$ is:

if $s$ is a leaf of the tree and $L(s)$ is a fully expanded propositional tableau, then for each formula of the form $\neg K_{i} \psi \in L(s)$, let $L^{\prime \prime}(s, \psi)=\left\{K_{i} \psi^{\prime}: K_{i} \psi^{\prime} \in L(s)\right\} \cup$ 
$\{\neg \psi\}$. If there is no ancestor $s^{\prime \prime}$ in the tree such that $L\left(s^{\prime \prime}\right)=L(s, \psi)$, then create an $i$-successor $s^{\prime}$ with $L\left(s^{\prime}\right)=L^{\prime \prime}(s, \psi)$.

and step $2(\mathrm{c})$ for $5_{n}$ is:

if $s$ is a leaf of the tree and $L(s)$ is a fully expanded propositional tableau, then for each formula of the form $\neg K_{i} \psi \in L(s)$, let $L^{\prime \prime}(s, \psi)=\left\{K_{i} \psi^{\prime}: K_{i} \psi^{\prime} \in L(s)\right\} \cup$ $\left\{\neg K_{i} \psi^{\prime}: \neg K_{i} \psi^{\prime} \in L(s)\right\} \cup\{\neg \psi\}$. If there is no ancestor $s^{\prime \prime}$ in the tree such that $L\left(s^{\prime \prime}\right)=L(s, \psi)$, then create an $i$-successor $s^{\prime}$ with $L\left(s^{\prime}\right)=L^{\prime \prime}(s, \psi)$.

This modification ensures that (4) and (5) conditions in the definition of tableaux are satisfied while construction terminates and the depth of the constructed pretableau tree is polynomial in $\left|\phi_{0}\right|^{2}$. This tree can be traversed in depth-first fashion while using space polynomial in $\left|\phi_{0}\right|$, since $|L(s)| \leq 2\left|\phi_{0}\right|$ for any $s$.

Finally, we can extend the algorithm of Halpern and Moses (1992) to $\mathscr{L}_{n}^{C, R, K, X, A}(\Phi)$ formulas. The extended algorithm constructs a tableau $T=\left(S, L, \mathscr{P} \mathscr{L}, \mathscr{K}_{1}, \ldots, \mathscr{K}_{n}\right.$, $\left.\mathscr{A}_{1}, \ldots, \mathscr{A}_{n}\right)$ which is the same as a tableau for $K_{n}^{Z}$, but with awareness sets and language assignment function added. We show how to extend the step for forming a fully expanded propositional tableau by expansion rules for formulas of the form $A_{i} \psi, C_{i}$ and $R_{i j}$ in such a way that information about every node in the tableau can still be stored using space polynomial in $|\phi|$ (the formula for which we are constructing a tableau) and the number of agents $n$. The modal depth of the tableau is not affected. Then we add additional conditions for when a node is marked as unsatisfiable. Finally, we show that for every formula $\psi, \psi \in L(s)$ implies $T, s \models \psi$ and $\neg \psi \in L(s)$ implies $T, s \models \neg \psi$, where $T$ is the model corresponding to the tableau, $s$ is a node marked as satisfiable, and $L(s)$ is its labelling.

The additional expansion rules are:

rel-awareness $\quad$ if $A_{i} \psi, R_{i j} \in L(s)$, then create a successor $s^{\prime}$ of $s$ with $L\left(s^{\prime}\right)=L(s) \cup$ $\left\{A_{j} \psi\right\}$

transitivity if $R_{i j}, R_{j k} \in L(s)$, then create a successor $s^{\prime}$ of $s$ with $L\left(s^{\prime}\right)=L(s) \cup$ $\left\{R_{i k}\right\}$

full-awareness $\quad$ if $R_{i j}, C_{i} \in L(s)$, then create a successor $s^{\prime}$ of $s$ with $L\left(s^{\prime}\right)=L(s) \cup$ $\left\{C_{j}\right\}$

The $i$-expansion step 2(c) is modified to include awareness formulas:

Creating $i$-successor nodes: if $s$ is a leaf of the tree, $L(s)$ is not blatantly inconsistent, and $L(s)$ is a fully expanded propositional tableau, then for each formula of the form $\neg K_{i} \psi \in L(s)$ create an $i$-successor node $s^{\prime}$ (add an edge from $s$ to $s^{\prime}$ to the tree labelled $i$ ) and let $L\left(s^{\prime}\right)=\left\{\psi^{\prime}: K_{i} \psi^{\prime} \in L(s)\right\} \cup\left\{A_{i} \psi^{\prime}: A_{i} \psi^{\prime} \in L(s)\right\} \cup$ $\left\{\neg A_{i} \psi^{\prime}: \neg A_{i} \psi^{\prime} \in L(s)\right\} \cup\{\neg \psi\}$.

Additional conditions for when a node is marked as unsatisfiable are:

mark $s$ as unsatisfiable if $L(s)$ contains $\neg A_{i} \psi$ where $\Phi(\psi) \subseteq \Phi\left(\left\{\psi^{\prime}: A_{i} \psi^{\prime} \in\right.\right.$ $L(s)\})$

mark $s$ as unsatisfiable if $L(s)$ contains $\neg R_{i i}$ for any $i$

mark $s$ as unsatisfiable if $L(s)$ contains $C_{i}$ and $\neg A_{i} \psi$ for any $i$ and $\psi$. 
Note that to store the node information in the extended language it is not enough to have a bit vector of length $2|\phi|$ to represent which of $\phi$ 's subformulae or their negations are present, but we also need $n|\phi|$ bits to represent extra formulas which may be added by step rel-awareness, $2 n^{2}$ bits for the formulas of the form $R_{i j}$ added by transitivity and $2 n$ for the formulas of the form $C_{i}$ which may be added by fullawareness. However, the resulting space usage is still polynomial in $|\phi|$ and $n$ (or in $|\phi|$ if we are treating $n$ as a constant).

Finally, we need to show that if a node $s$ is marked as satisfiable, then we can construct a language assignment function $\mathscr{P} \mathscr{L}$ and awareness sets $\mathscr{A}_{1}(s), \ldots, \mathscr{A}_{n}(s)$ so that for all formulas $\psi \in \operatorname{Subf}(\phi)$ of the form $A_{i} \gamma, C_{i}, R_{i j}$,

$$
\psi \in L(s) \text { implies } T, s \models \psi \text {, and } \neg \psi \in L(s) \text { implies } T, s \models \neg \psi \text {. }
$$

Instead of constructing $\mathscr{A}_{i}(s)$ directly, we construct their propositional subsets $\mathscr{A}_{i}^{p}(s)$. The construction is similar to the one in the proof of Theorem 3. We use fresh propositional witnesses $q_{1}, \ldots, q_{n}, \ldots$ which are not in $\operatorname{Subf}(\phi)$. For each agent $i$, let $X_{i}^{0}(s)$ to $\left\{p \in \Phi(\psi): A_{i} \psi \in L(s)\right\}$. We set $\mathscr{P} \mathscr{L}^{0}(s)$ to be $\cup_{i} X_{i}^{0}(s)$. If $C_{i} \in L(s)$, we reassign $X_{i}^{0}(s)$ to be $\mathscr{P} \mathscr{L}^{0}(s)$.

Next, we construct awareness sets and language assignments that work locally at each $s$. (After that, we will need to make sure that the condition $k a$ is satisfied).

The construction of 'locally correct' $\mathscr{P} \mathscr{L}(s)$ and $\mathscr{A}_{i}^{p}(s)$ consists of $n+1$ steps, where $n$ is the number of agents. At the step corresponding to agent $i$, if $R_{i k}, \neg R_{i j} \in$ $L(s)$ then we add $q_{i}$ to $X_{k}^{i}$, for every such $k$, and to $X_{i}^{i}$. We also add $q_{i}$ to $\mathscr{P} \mathscr{L}^{i}(s)$ and reassign $X_{m}^{i}(s)$ to be $\mathscr{P} \mathscr{L}^{i}(s)$ for all $m$ such that $C_{m} \in L(s)$. Finally, we set $\mathscr{P} \mathscr{L}^{n+1}(s)$ to be $\mathscr{P} \mathscr{L}^{n}(s) \cup\left\{q_{n+1}\right\}$ and set $X_{m}^{n+1}(s)$ to be $\mathscr{P} \mathscr{L}^{n+1}(s)$ for all $m$ such that $C_{m} \in L(s)$. (The latter step is to deal with the situation when for some $m$, $X_{m}^{n}(s)=\mathscr{P} \mathscr{L}^{n}(s)$ but $\neg C_{m} \in L(s)$.) We set $\mathscr{A}_{i}^{p}(s)$ to be $X_{i}^{n+1}$.

It is straighforward to check that the construction above ensures that for subformulas of $\phi$, if $A_{i} \psi$ is in $L(s)$ then its propositional variables are in $\mathscr{A}_{i}^{p}(s)$, and if $\neg A_{i} \psi \in L(s)$ then there is at least one propositional variable in $\psi$ that is not in $\mathscr{A}_{i}^{p}(s)$. Also, the following conditions hold:

(R) if $R_{i j} \in L(s)$, then $\mathscr{A}_{i}^{p}(s) \subseteq \mathscr{A}_{j}^{p}(s)$

(notR) if $\neg R_{i j} \in L(s)$, there is a propositional variable that is in $\mathscr{A}_{i}^{p}(s)$ but not in $\mathscr{A}_{j}^{p}(s)$,

(C) if $C_{i} \in L(s)$ then $\mathscr{A}_{i}^{p}(s)=\mathscr{P} \mathscr{L}(s)$

(notC) if $\neg C_{i} \in L(s)$ then $\mathscr{A}_{i}^{p}(s) \neq \mathscr{P} \mathscr{L}(s)$.

However the condition $k a$ is not guaranteed to hold. Although for all $s$ and $t$ with $(s, t) \in \mathscr{K}_{i}$, the set of formulas of the form $A_{i} \psi$ and $\neg A_{i} \psi$ in $L(s)$ and $L(t)$ are the same, formulas of the form $R_{i j}$ and $C_{i}$ may be different. For example, it is possible that $R_{i j} \in L(s)$ and $\neg R_{i j} \in L(t)$, and similarly for $C_{i} \in L(s)$ and $\neg C_{i} \in L(t)$. This may lead to $\mathscr{A}_{i}^{p}(s)$ being different from $\mathscr{A}_{i}^{p}(t)$.

As in the proof of Theorem 3, for a node $s$ let $K(s, i)$ be the set of $i$-descendants of $s$ (nodes in the reflexive transitive closure of the $i$-successor relation). Let $A(s, i)$ be $\bigcup_{t \in K(s, i)} \mathscr{A}_{i}^{p}(t)$. We start with the root node $s_{0}$ of the tableaux and for each $i$ and $t \in K\left(s_{0}, i\right)$ make $\mathscr{A}_{i}^{p}(t)$ equal to $A\left(s_{0}, i\right)$. This involves adding extra propositional 
variables to $\mathscr{A}_{i}^{p}(t)$ which are then propagated to $\mathscr{A}_{j}^{p}(t)$ for all $j$ such that $R_{i j} \in L(t)$ or $C_{j} \in L(t)$, and added to $\mathscr{P} \mathscr{L}(t)$ if not already there. This ensures that conditions (R) and (C) are satisfied. Then we check for every $\neg R_{k m}$ and $\neg C_{k} \in L(t)$ whether (notR) and (notC) are still satisfied and if not add a new witness proposition to $\mathscr{A}_{k}^{p}(t)$ (and propagate it to ensure (R) and (C)) and to $\mathscr{P} \mathscr{L}(t)$ (and propagate it to $\mathscr{A}^{p}(m)$ with $C_{m} \in L(t)$ ). Similarly to the proof of Theorem 3, it can be proved that the fixes for (notR) and (notC) do not affect $\mathscr{A}_{i}^{p}(t)$. After we execute this procedure once for each agent $i, \mathscr{A}_{i}^{p}\left(s_{0}\right)$ may be changed for some $i$ which requires fixing $k a$ the second time. However the second time none of the problems with (notR) and (notC) can occur, so there is no change to any $\mathscr{A}_{j}^{p}\left(s_{0}\right)$ as the result. After two passes, the procedure terminates and results in a set of awareness sets and language assignments that satisfy $k a$ and $(\mathrm{R}),(\mathrm{C}),(\operatorname{notR})$ and (notC).

Now we prove that $\psi \in L(s)$ implies $T, s \models \psi$, and $\neg \psi \in L(s)$ implies $T, s \models \neg \psi$. Consider the three non-trivial cases:

$\psi=A_{i} \gamma$. If $A_{i} \gamma \in L(s)$, then $\Phi(\gamma) \subseteq \mathscr{A}_{i}^{p}(s)$,so $T, s=A_{i} \gamma$. If $\neg A_{i} \gamma \in L(s)$, then $\mathscr{A}_{i}^{p}(s)$ is not equal to $\mathscr{P} \mathscr{L}(s)$ (because $s$ is consistent, so $\left.C_{i} \notin L(s)\right)$ and $\mathscr{A}_{i}(s)$ does not contain some variable from $\gamma$ (because again due to consistency $\Phi(\gamma) \nsubseteq$ $\left.\mathscr{A}_{i}^{p}(s)\right)$. So $T, s \models \neg A_{i} \gamma$.

$\psi=C_{i}$. If $C_{i} \in L(s)$, then $\mathscr{A}_{i}^{p}(s)=\mathscr{P} \mathscr{L}(s)$, so $T, s=C_{i}$. If $\neg C_{i} \in L(s)$ then $\mathscr{A}_{i}^{p}(s) \neq \mathscr{P} \mathscr{L}(s)$, so $T, s \models \neg C_{i}$.

$\psi=R_{i j}$. Suppose by contradiction that $R_{i j} \in L(s)$ and $\mathscr{A}_{i}(s) \nsubseteq \mathscr{A}_{j}(s)$. By construction and consistency of $s, \mathscr{A}_{i}(s)$ and $\mathscr{A}_{j}(s)$ are not equal to $\mathscr{P} \mathscr{L}(s)$. So the variable which is in $\mathscr{A}_{i}(s)$ but not in $\mathscr{A}_{j}(s)$ is either some $p$ such that $p$ occurs in $\gamma$ and $A_{i} \gamma \in L(s)$, or one of the witness variables. The first case is excluded by the rel-awareness rule which forces $A_{j} \gamma \in L(s)$, hence in $p \in \mathscr{A}_{j}(s)$. The second case is excluded by the transitivity rule and the way we add witnesses.

Let $\neg R_{i j} \in L(s)$. Then we added a witness $q_{i}$ to $X_{i}^{i}$, which is not in $\mathscr{A}_{j}(s)$. So $T, s \mid=\neg R_{i j}$.

\section{Discussion}

We cannot directly compare our logic with HR13 logic since we have chosen a different truth definition (a formula does not have to belong to the language defined in a state in order to be true in that state). This means that our definition of validity is different, too.

There is a clear sense however that $R_{i j}$ closely corresponds to $\forall x\left(A_{i} x \rightarrow A_{j} x\right)$ and $C_{i}$ corresponds to $\forall x A_{i} x$, and in this sense we can reason about a strict subset of the properties HR13 can reason about. The main advantage of our approach is the decidability of the logic. This means that similarly to the logic of general awareness it can be used for automated reasoning and verification of multi-agent systems. 
Our approach can express properties of relative awareness; however it is not expressive enough to state properties of relative explicit knowledge rather than awareness. As pointed out to us by Yoram Moses, it is easy to paraphrase Example 1 so that it is no longer expressible in $\mathscr{L}_{n}^{C, R, K, X, A}(\Phi)$. Namely, consider replacing the last sentence in Example 1 by the following sentence

Example 5.

... On the other hand, the investor explicitly knows that there is something that the broker explicitly knows but he is not aware of.

(replace 'the broker is aware of' with 'the broker explicitly knows'). This example can be expressed in the language of HR13 logic but not in $\mathscr{L}_{n}^{C, R, K, X, A}(\Phi)$.

\section{Conclusions}

We have pointed out that the full expressiveness of unrestricted quantification over formulas is not needed to express knowledge of unawareness in the motivating examples of Halpern and Rêgo (2006, 2009b), that quantification restricted to full and relative awareness is sufficient, and that the logic of full and relative awareness is decidable (in PSPACE). We have presented a sound and complete axiomatisation of that logic.

By negating full and relative awareness, we have seen that we can express the fact that there is at least one fact the agent is not aware of, and there is at least one fact the agent is aware of and the other agent is not aware of, respectively. This could possibly be generalised to there is at least $n$, for arbitrary natural numbers $n$. We studied such "at least $n$ " operators in (Ågotnes and Alechina, 2006), where we investigated an epistemic language interpreted in purely syntactic structures (Fagin et al., 1995), extended with an operator $\min (n)$ meaning that the agent explicitly knows at least $n$ formulae. A promising direction of research would be to introduce relative knowledge operators to express examples such as 'The investor explicitly knows that there is something that the broker explicitly knows but the investor is not aware of'. Perhaps relative knowledge can be expressed using formulas to express inclusion of accessibility relations in the states building on the work of van Ditmarsch et al. (2009).

\section{References}

Thomas Ågotnes and Natasha Alechina. Full and relative awareness: A decidable logic for reasoning about knowledge of unawareness. In Dov Samet, editor, Theoretical Aspects of Rationality and Knowledge: Proceedings of the 11th conference (TARK 2007), pages 6-14. UCL Presses Universitaires de Louvain, 2007. ISBN 978-2-8746-3077-4. 
Thomas Ågotnes and Natasha Alechina. Knowing minimum/maximum $\mathrm{n}$ formulae. In Gerhard Brewka, Silvia Coradeschi, Anna Perini, and Paolo Traverso, editors, Proceedings of the 17th European Conference on Artificial Intelligence (ECAI 2006), pages 317-321. IOS Press, 2006.

Oliver Board and Kim-Sau Chung. Object-based unawareness. In Giacomo Bonanno, Wiebe van der Hoek, and Michael Wooldridge, editors, Proceedings of The 7th Conference on Logic and the Foundations of Game and Decision Theory (LOFT), pages 35-41, July 2006.

Ronald Fagin and Joseph Y. Halpern. Belief, awareness and limited reasoning. Artificial Intelligence, 34:39-76, 1988.

Ronald Fagin, Joseph Y. Halpern, Yoram Moses, and Moshe Y. Vardi. Reasoning About Knowledge. The MIT Press, Cambridge, Massachusetts, 1995.

Joseph Y. Halpern. Alternative semantics for unawareness. Games and Economic Behaviour, 37:321-339, 2001.

Joseph Y. Halpern and Yoram Moses. A guide to completeness and complexity for modal logics of knowledge and belief. Artificial Intelligence, 54(2):319-379, 1992.

Joseph Y. Halpern and Leandro Chaves Rêgo. Reasoning about knowledge of unawareness revisited. In Aviad Heifetz, editor, Proceedings of the 12th Conference on Theoretical Aspects of Rationality and Knowledge (TARK-2009), Stanford, CA, USA, July 6-8, 2009, pages 166-173, 2009a.

Joseph Y. Halpern and Leandro Chaves Rêgo. Reasoning about knowledge of unawareness revisited. Mathematical Social Sciences, 65(2):73-84, 2013.

Joseph Y. Halpern and Leandro Chaves Rêgo. Reasoning about knowledge of unawareness. In Principles of Knowledge Representation and Reasoning: Proceedings of the Tenth International Conference (KR'06), pages 6-13, Lake District, UK, June 2006. AAAI.

Joseph Y. Halpern and Leandro Chaves Rêgo. Reasoning about knowledge of unawareness. Games and Economic Behavior, 67(2):503 - 525, 2009b. doi: http://dx.doi.org/10.1016/j.geb.2009.02.001.

Aviad Heifetz, Martin Meier, and Burkhard Schipper. Interactive unawareness. Journal of Economic Theory, 2007. Forthcoming.

Salvatore Modica and Aldo Rustichini. Awareness and partitional information structures. Theory and Decision, 37:107-124, 1994.

Salvatore Modica and Aldo Rustichini. Unawareness and partitional information structures. Games and Economic Behaviour, 27:265-298, 1999.

Giacomo Sillari. Models of awareness. In Giacomo Bonanno, Wiebe van der Hoek, and Michael Wooldridge, editors, Proceedings of The 7th Conference on Logic and the Foundations of Game and Decision Theory (LOFT), pages 209-218, July 2006.

Hans P. van Ditmarsch, Wiebe van der Hoek, and Barteld P. Kooi. Knowing more - from global to local correspondence. In Craig Boutilier, editor, IJCAI 2009, Proceedings of the 21st International Joint Conference on Artificial Intelligence, Pasadena, California, USA, July 11-17, 2009, pages 955-960, 2009. 